\title{
Les portfolios européens des langues
}

\section{Christine Tagliante et Jacqueline Deleuse}

\section{(2) OpenEdition}

\section{Journals}

Édition électronique

URL : https://journals.openedition.org/ries/1979

DOI : $10.4000 /$ ries. 1979

ISSN : 2261-4265

\section{Éditeur}

France Education international

\section{Édition imprimée}

Date de publication : 1 décembre 2002

Pagination : 138-142

ISBN : 978-2854-2-0555-8

ISSN : 1254-4590

\section{Référence électronique}

Christine Tagliante et Jacqueline Deleuse, «Les portfolios européens des langues », Revue internationale d'éducation de Sèvres [En ligne], 31 | décembre 2002, mis en ligne le 25 novembre 2011, consulté le 08 juillet 2021. URL : http://journals.openedition.org/ries/1979 ; DOI : https://doi.org/ 10.4000/ries. 1979

Ce document a été généré automatiquement le 8 juillet 2021.

(ㄷ) Tous droits réservés 


\title{
Les portfolios européens des langues
}

\author{
Christine Tagliante et Jacqueline Deleuse
}

\section{La finalité, les objectifs}

1 Les portfolios européens des langues (PEL) sont issus d'un projet du Conseil de l'Europe, division des langues vivantes. Leur finalité est inscrite dans la recommandation $\mathrm{n}^{\circ} \mathrm{R}(82) 18$ du comité des ministres aux États membres :

- «le riche patrimoine constitué par les diverses langues et cultures de l'Europe est une ressource commune précieuse qui doit être protégée et développée ;

- un effort éducatif de grande ampleur est nécessaire pour faire de cette diversité non plus un obstacle à la communication mais une source de compréhension et d'enrichissement mutuels ;

- seule une meilleure connaissance des langues vivantes européennes pourra faciliter la communication et l'interaction entre Européens de langues différentes afin de promouvoir la mobilité, la compréhension mutuelle et la coopération et de surmonter les préjugés et la discrimination en Europe. »

Dans sa recommandation $n^{\circ} R$ (98) 6, le comité des ministres « encourage, entre autres mesures, le développement et l'utilisation par les apprenants d'un document personnel (portfolio européen des langues) dans lequel ils pourraient inscrire leurs qualifications et autres expériences linguistiques et culturelles significatives de manière transparente au plan international, afin d'étendre et de diversifier l'apprentissage des langues à tous les niveaux et tout au long de la vie.»

\section{Le concept, la philosophie}

Les objectifs que le portfolio européen des langues cherche à atteindre reflètent ceux du Conseil de l'Europe. Le symposium de Rüschlikon, en 1998 (Transparence et cohérence dans l'apprentissage des langues en Europe : objectifs, évaluation, certification), a jeté les bases 
de ce que pourrait être un outil permettant d'y répondre. Cet outil devrait se présenter sous la forme d'un document individuel visant à permettre à tous les Européens, dès le plus jeune âge, de devenir des citoyens plurilingues disposant de compétences interculturelles.

Cet objectif général permettrait notamment d'approfondir la compréhension mutuelle des citoyens en Europe dans le respect et la protection des diversités culturelles et des modes de vie; de permettre la poursuite de l'apprentissage des langues tout au long de la vie; de contribuer au développement de l'autonomie de l'apprenant en langues; de promouvoir la cohérence et la transparence des programmes de langues; de décrire clairement les qualifications pour faciliter la mobilité.

\section{Les principes}

Pour promouvoir ces objectifs, le PEL se base sur les principes suivants :

- toutes les compétences en langues ainsi que les connaissances culturelles doivent être valorisées, qu'elles aient été acquises à l'intérieur ou à l'extérieur du système d'éducation formelle :

- le PEL est la propriété de l'apprenant et est basé sur son auto-évaluation combinée avec l'évaluation par des professionnels ;

- le PEL a deux fonctions: une fonction pédagogique («processus») et une fonction de présentation/documentation ("produit»);

- les niveaux de compétences en langues sont ceux décrits dans le Cadre européen commun de référence.

\section{Les points communs à tous les portfolios européens des langues}

6 À ce jour, trente modèles de PEL ont été validés par le Comité européen de validation et de nombreux autres sont en cours d'élaboration et d'expérimentation. Ces différents modèles présentent des maquettes sensiblement différentes mais ont tous un air de famille qui en font un document reconnaissable à travers l'Europe.

7 Tous les PEL comportent trois parties, plus ou moins développées selon l'âge de l'apprenant, ses besoins particuliers et son contexte éducatif :

un passeport de langues, dont la maquette est commune à tous les pays participants, mentionne les certifications officielles obtenues, fait état des compétences langagières et interculturelles significatives mais non diplômantes et réserve une place à l'autoévaluation ainsi qu'à l'évaluation institutionnelle ;

une biographie langagière, basée sur les niveaux du Cadre européen commun de référence, donne à l'apprenant l'occasion d'estimer ses compétences et de planifier, gérer, son apprentissage; elle permet cette estimation dans plusieurs langues, favorisant ainsi la promotion du plurilinguisme.

un dossier offre à l'apprenant la possibilité de sélectionner et de réunir les documents qu'il considère comme étant des preuves de ses compétences langagières et interculturelles. 


\section{Les perspectives}

11 Depuis le symposium de 1991, qui a lancé le Cadre européen commun de référence et le portfolio, une demande croissante de cohérence et de transparence dans la validation des compétences linguistiques se manifeste un peu partout en Europe dans les États membres. Le Cadre européen commun, qui présente une échelle de compétences en six niveaux, devrait être l'outil avec lequel on tentera d'harmoniser les certifications.

12 Le défi des années à venir est donc d'établir un lien concret entre les examens et les tests de langues et le Cadre européen. Vaste et passionnant programme, qui rejoint la finalité des portfolios: "promouvoir la mobilité, la compréhension mutuelle et la coopération et surmonter les préjugés et la discrimination en Europe. »

Christine Tagliante

\section{Témoignages : Il mio Primo Portfolio delle lingue (PPL) et l'Europäisches Portfolio der Sprachen (EPS)}

13 À l'initiative de l'Association européenne des enseignants (AEDE) ${ }^{1}$ et sous l'égide du ministère des Affaires européennes, une double visite a été effectuée en Italie, dans la province de Turin, ainsi qu'en Allemagne à Kleve et Soest en Rhénanie du NordWestphalie, pour y observer la mise en œuvre du portfolio dans des classes du primaire et du secondaire.

Il mio Primo Portfolio delle lingue s'inspirant de Mon premier portfolio élaboré par le CIEP, validé par le Conseil de l'Europe le 2 mars 2002, s'adresse à des élèves de l'école primaire de huit à onze ans.

15 L'Europäisches Portfolio der Sprachen concerne des élèves du secondaire de dix à dixneuf ans. Comme l'Italie, l'Allemagne a participé à l'expérimentation internationale et nationale (30 000 élèves de 1997 à 2000), qui applique les principes du Cadre européen commun de référence. Il se compose comme le PPL de trois parties (biographie, dossier, passeport ${ }^{2}$ ) et s'adresse directement à l'élève pour le guider dans l'apprentissage de son auto-évaluation (cinq domaines de compétence, six niveaux) et du plurilinguisme.

16 Ce portfolio, dans le primaire comme dans le secondaire, en Italie comme en Allemagne, introduit une pédagogie innovante dont pourraient à plus d'un titre s'inspirer nos classes de langues en France.

\section{Le plaisir d'apprendre et de communiquer}

17 Avec le portfolio, l'extérieur pénètre dans la salle de classe. Des objets choisis par les élèves de l'école primaire italienne de Susa dans des classes d'anglais et de français (boîtes de fromages français, bonbons anglais, billets de métro londoniens, etc.) sont conservés dans une boîte-dossier, listés et catalogués. Ils sont déclencheurs de recherches, d'échanges, de communication dans la langue cible. L'école ne privilégie pas les seules expériences de formulation langagière, mais fait trouver les usages et les plaisirs que l'élève de cet âge souhaite rencontrer. L'objectif n'est pas de produire des énoncés formels, mais que les mots retrouvent leur vrai fonction d'interaction dans des activités quotidiennes avec une implication sociale immédiate.

Dans la Realschule de $\mathrm{Kleve}^{3}$, la pièce de théâtre jouée les jours précédents en néerlandais devient aussi objet d'analyse en allemand : les élèves doivent, en binôme, 
retrouver les compétences langagières qu'ils ont utilisées en tant qu'acteurs. Le savoir culturel sert de matériau linguistique alors qu'il est souvent, dans un cours traditionnel, l'objet d'une programmation artificielle que l'élève n'est pas prêt à recevoir.

\section{Vers une pédagogie de l'échange plurilingue}

Il ne s'agit pas d'échange au sens traditionnel du terme, mais d'une véritable coopération pédagogique à distance entre classes utilisant le PPL.

Les élèves de l'école de Susa, étudiant le français, travaillent sur un projet commun annuel avec des classes du Val d'Aoste. Ce projet, bâti sur le passé romain de Susa, culmine avec la visite des élèves francophones et une évaluation générale de ce passé historique en s'appuyant sur la biographie langagière. Dans un autre cours qui réunit une classe d'anglais et de français, un dialogue s'instaure dans les deux langues entre les élèves et leurs professeurs ${ }^{4}$ qui posent des questions sur le passé de Susa. Les réponses se font en français ou en anglais, selon la langue étudiée par les élèves. Sans faire le détour par leur langue maternelle, ils s'efforcent de comprendre l'une et l'autre et de réagir dans la langue d'apprentissage.

À la Realschule de Kleve, les enseignants allemands et néerlandais intensifient leur coopération pour développer de véritables situations de communication en classe, et susciter de façon naturelle l'utilisation des deux langues dans les classes de part et d'autre de la frontière.

\section{Former les élèves à l'autoévaluation}

À l'école Pieta Gemelli ${ }^{5}$, les élèves ont appris à analyser les raisons pour lesquelles il est important de s'auto-évaluer en langues. Ils verbalisent avec aisance et écartent fermement sous-estimation et surestimation: «Siamo noi che ci conosciamo bene dentro », « La maestra si accorge che non imparo, se non sono sincero »; « Ho maturato quella competenza. $»^{6}$. Autant de réflexions qui montrent que l'enfant peut dire comment il s'évalue et qui mettent en évidence l'investissement affectif face à une langue étrangère. Les parents ont reçu un minidossier expliquant les principes et les raisons de l'autoévaluation et les réticences du début ont cédé le pas à l'adhésion : plus de maturité chez l'enfant, enseignement plus diversifié et ouverture sur le monde.

À la Realschule de Kleve, en deuxième année de néerlandais, le professeur définit les compétences d'expression écrite, orale et de correction de la langue, puis il demande à chaque binôme de décrire en néerlandais des supports différenciés (carte postale, photo, etc.). Chacun d'entre eux lit les phrases qu'il a écrites. Les élèves sont ensuite invités à utiliser le portfolio, à repérer les descripteurs concernés pour s'autoévaluer. Intérêt du croisement des regards : le dialogue est ouvert entre l'évaluation de l'élève par lui-même, celle de ses camarades et celle de l'enseignant qui s'appuie sur son expérience, favorise la prise de conscience et confère une valeur plus objective à l'évaluation. 


\section{Former les enseignants à l'autoévaluation et construire des équipes pédagogiques européennes}

Cette révolution douce, mais profonde dans l'apprentissage des langues tout au long de la vie, dans l'Europe et dans le monde ne peut faire l'économie de la formation des enseignants. Ainsi le Centre de Soest en Allemagne, concepteur et éditeur de l'EPS, a accueilli quatre-vingt-quinze enseignants et directeurs de soixante-dix écoles (dix-neuf utilisent le portfolio dans toutes les langues), lors d'une formation de deux jours en présence d'experts des portfolios belges et néerlandais et d'un directeur d'entreprise import-export. Dans les ateliers, les participants ont élaboré du matériel didactique (cours pour introduire le portfolio, aides méthodologiques, argumentaire pour tous les publics) et ont échangé leurs expériences et leurs documents, afin de développer davantage encore leurs réseaux. Un guide pour les enseignants est en cours d'élaboration.

Une cinquantaine de professeurs d'écoles de la province de Turin ont reçu pendant quelques semaines une formation à l'utilisation du portfolio et à l'autoévaluation. Un guide pour l'enseignant a été élaboré par la direction générale régionale du Piémont.

Il est important de mentionner que les professeurs, en Allemagne comme en Italie, sont très demandeurs de réseaux portfolios, afin de créer une véritable synergie à travers les États membres.

\section{Perspectives}

Il faut songer très concrètement à développer des échanges d'enseignants et des partenariats PEL de classes et d'écoles, en particulier dans la cadre de la coopération entre les établissements ${ }^{7}$. Le PEL s'avèrerait être un outil fédérateur prenant plus d'importance à mesure que le plan d'action pour la mobilité se met en place ${ }^{8}$. Il pourrait également favoriser la synergie entre le primaire et le secondaire qui a du mal à s'instaurer. Il convient de souligner le rôle qu'une association comme l'AEDE pourrait jouer dans la diffusion de l'outil PEL dans les États membres en favorisant l'accès, même modestement, aux différentes expériences innovantes européennes ${ }^{9}$ et en jouant le rôle de véritable trait d'union entre les systèmes éducatifs.

Dans cette perspective, le portfolio semble être un outil de première importance en Europe en permettant un rapprochement organisé entre les jeunes générations par l'affectif et par les langues.

Jacqueline Deleuse

Danielle Lavollée

\section{NOTES}

1. L'AEDE compte plus de 20000 adhérents en Europe et regroupe des enseignants du primaire, du secondaire, du supérieur, des chercheurs, des inspecteurs. 
2. Les enseignants doivent y apposer un commentaire, leur signature et la date; l'école qui a apporté son aide et son concours est invitée à mettre son cachet.

3. Kleve est située à huit kilomètres de la frontière germano-hollandaise. À la Städtische Realschule an der Hoffmannallee, huit professeurs de néerlandais utilisent l'EPS à raison de dix séances par an en deuxième année de néerlandais LV2 où l'option devient obligatoire pour quatre ans avec quatre heures hebdomadaires. L'un d'entre eux qualifie le portfolio «d'enrichissant, motivant, complémentaire ».

4. Le professeur d'école de chacune des deux classes et les collègues enseignant l'anglais et le français.

5. École située dans un quartier populaire de Turin.

6. «On est seul à se connaître à l'intérieur de soi », « Ma maîtresse s'aperçoit que je ne dis pas la vérité ", « Cette compétence a mûri en moi. »

7. cf. L'enseignement des langues vivantes. Perspectives, Les actes de la DESCO, CNDP, 2001, et le BO de rentrée $\mathrm{N}^{\circ} 16$ du 18 avril 2002 sur L'Ouverture internationale et européenne.

8. Le Plan d'action pour la mobilité (2000) prévoit de développer la mobilité de toutes les personnes en formation et des enseignants en Europe.

9. Projet Scala proposé par les sections AEDE bulgares et suisses définissant un outil d'autoévaluation par rapport aux différents niveaux de compétence.

\section{INDEX}

Mots-clés : enseignement des langues, portfolio, Conseil de l'Europe

Index géographique : Europe

\section{AUTEURS}

\section{CHRISTINE TAGLIANTE}

Responsable du bureau de l'évaluation et des certifications au CIEP

\section{JACQUELINE DELEUSE}

Inspectrice honoraire d'anglais

Danielle Lavollée

Professeur-formateur en allemand, danielle.lavollee@wanadoo.fr 